\title{
Hiccup Due to Aripiprazole Plus Methylphenidate Treatment in an Adolescent with Attention Deficit and Hyperactivity Disorder and Conduct Disorder: A Case Report
}

\author{
Meryem Ozlem Kutuk ${ }^{1}$, Gulen Guler ${ }^{2}$, Ali Evren Tufan ${ }^{3}$, Ozgur Kutuk ${ }^{4}$ \\ ${ }^{1}$ Department of Child and Adolescent Psychiatry, ${ }^{4}$ Department of Medical Genetics, Adana Dr. Turgut Noyan Medical and Research Center, \\ Baskent University School of Medicine, Adana, ${ }^{2}$ Department of Child and Adolescent Psychiatry, Elazig Mental Health Hospital, Elazig, \\ ${ }^{3}$ Department of Child and Adolescent Psychiatry, Abant Izzet Baysal University School of Medicine, Bolu, Turkey
}

\begin{abstract}
Our case had hiccups arising in an adolescent with the attention deficit and hyperactivity disorder (ADHD) and conduct disorder (CD) after adding aripiprazole treatment to extended-release methylphenidate. Actually, antipsychotics are also used in the treatment of hiccups, but studies suggest that they can cause hiccups as well. Within 12 hours of taking 2.5 mg aripiprazole added to extended-release methylphenidate at a dose of $54 \mathrm{mg} /$ day, 16-year-old boy began having hiccups in the morning, which lasted after 3-4 hours. As a result, aripiprazole was discontinued and methylphenidate was continued alone because we could not convince the patient to use another additional drug due to this side effect. Subsequently, when his behavior got worsened day by day, his mother administered aripiprazole alone again at the dose of $2.5 \mathrm{mg} /$ day at the weekend and continued treatment because hiccup did not occur again. But when it was administered with methylphenidate on Monday, hiccup started again next morning and lasted one hour at this time. In conclusion, we concluded that concurrent use of methylphenidate and aripiprazole in this adolescent led to hiccups.
\end{abstract}

KEY WORDS: Aripiprazole; Cytochrome P-450 CYP2D6; Hiccup; Methylphenidate.

\section{INTRODUCTION}

Hiccup is a repetitive, involuntary, spasmodic and characteristic voice that can occur with closure of the glottis suddenly as a result of involuntary contraction of the diaphragm and respiratory muscles. ${ }^{1)}$ Though the pathophysiological processes that lead to hiccups have not been completely established, central nervous system disorders, gastrointestinal disorders and tumors, metabolic and electrolyte disorders and various drugs are implicated as causes of hiccup. ${ }^{1)}$ In drug-induced hiccups, aripiprazole is very often incriminated for transient and persistent hiccups among adult and adolescent patients. ${ }^{2,3)}$ Here, we present a case of hiccups arising in adolescent with an extensive metabolizer genotype for CYP2D6 when using aripiprazole in addition to extended-release methylpheni-

Received: July 21, 2016 / Accepted: August 18, 2016

Address for correspondence: Gulen Guler, MD

Department of Child and Adolescent Psychiatry, Elazig Mental

Health Hospital, Rizaiye Mah. Kazım Hoca Street. No: 23/B, Elazig 23100, Turkey

Tel: +90-424-238-79-53, Fax: +90-424-212-78-31

E-mail: dr.gulen@hotmail.com date. Of note, his hiccups ceased when aripiprazole was used without methylphenidate.

\section{CASE}

The patient was a 16-year-old boy who was referred to the outpatient clinic with complaints of behavioral problems like difficulty in anger control, running away from school and home, smoking and telling lies. In his medical history, he was diagnosed with the attention deficit and hyperactivity disorder (ADHD) 5 years ago by a child and adolescent psychiatrist and received methylphenidate 54 $\mathrm{mg} /$ day alone, with significant benefit for 5 years. But, within the last year, he started to fight, tell lies, smoke and also to be a school truant. Finally, it was learned that he took his father's car without permission and had an accident with it. His family told that he did not have attention deficit problems during methylphenidate treatment and his academic achievement was satisfactory. In addition, he did not want to use methylphenidate anymore. In the psychiatric evaluation, he did not have eye contact and he told that he did not want to talk. He seemed very nervous.

(c) This is an Open-Access article distributed under the terms of the Creative Commons Attribution Non-Commercial License (http://creativecommons.org/licenses/by-nc/4.0) which permits unrestricted non-commercial use, distribution, and reproduction in any medium, provided the original work is properly cited. 
And after ten minutes in silence, he shut the door hard and went out.

According to psychiatric assessment, he was diagnosed with ADHD and conduct disorder (CD) according to Diagnostic and Statistical Manual of Mental Disorders, fifth edition (DSM 5) ${ }^{4)}$ and was prescribed aripiprazole $2.5 \mathrm{mg} /$ day adding to extended-release methylphenidate at a dose of $54 \mathrm{mg} /$ day. Next day, he had an episode of hiccups in the morning starting 12 hours after the administration of first dose of $2.5 \mathrm{mg}$ /day aripiprazole at night and hiccups lasted for 4 hours, which led him to come to clinic in great concern. He was extremely disturbed from this side effect. Therefore, we were unable to convince the patient to use another drug for behavioral problems. Owing to this side effect, it was thought that he may be a poor or intermediate metabolizer and pharmacogenetic testing was requested from medical genetics laboratory for CYP2D6.

While waiting for test results, behavioral problems of the case were aggravated and his mother gave aripiprazole again at the dose of $2.5 \mathrm{mg}$ /day for 2 weekend days saying that it was a vitamin for his lack of appetite, due to methylphenidate because he had drug holidays for methylphenidate every weekend. She told that she observed no hiccup with only aripiprazole but when she gave it again in weekdays in combination with methylphenidate, the hiccup started 12 hours later again and lasted for an hour at this time. She thought that he was better with aripiprazole when administered at alone, so at present patient is on only aripiprazole $5 \mathrm{mg}$ /day as the schools are closed, and no hiccup is observed. Moreover, his behavioral problems decreased markedly. Meanwhile, our case was genotyped as extensive/normal metabolizer for CYP2D6. Evaluation with the Naranjo algorithm revealed a score of 7 (probably adverse drug reaction). ${ }^{5)}$

\section{DISCUSSION}

Here, we present a case of hiccups arising in a CYP2D6 extensive/normal metabolizer adolescent who was using aripiprazole plus extended-release methylphenidate for the treatment of ADHD and CD. The use of atypical antipsychotics concomitantly with stimulants in children and adolescents is becoming more common as clinicians try to treat comorbidities involving $\mathrm{ADHD}, \mathrm{CD}$, oppositional defiant disorder, mental retardation and pediatric bipolar disorder despite their potentially opposing pharmacological mechanism. ${ }^{6,7)}$

Previous reports of hiccups with aripiprazole exist in the literature, ${ }^{2,3)}$ but our case is of interest in that a drug used commonly in child psychiatry such as methylphenidate promoted aripiprazole to cause hiccup. Although the precise roles of neurotransmitters within the reflex arc of hiccups remain to be completely understood, current data suggest that both dopamine and serotonin may play roles in the development of hiccups. Methylphenidate is currently the most commonly prescribed agent used to treat ADHD and it is thought to exert its effects by inhibiting dopamine and norepinephrine reuptake and by binding the serotonin transporter. ${ }^{8,9)}$ It is also used for the treatment of hiccup in patients. ${ }^{10,11)}$

Furthermore, aripiprazole, which is a partial agonist of dopamine 2,3 and 5-HT1A receptors, is used to stabilize dopamine and serotonin systems. Previous reports stated that both hypo- and hyper-dopaminergic states may give rise to hiccups and both drugs can lead to these conditions in our case. ${ }^{2,3)}$

The members of the cytochrome $\mathrm{P} 450$ protein family mediate the metabolism of many pharmaceutical agents and genetic variability in these enzymes may dictate a patient's response to a drug. Identification of genotypes of these enzymes can minimize adverse drug reactions and improve patients' response. CYP2D6 is the main cytochrome P450 enzyme involved in the metabolism of antipsychotics and antidepressants. ${ }^{12)}$ In our case, we utilized PGX-CYP2D6 Strip Assay (Vienna Lab Diagnostics, Vienna, Austria) to determine the genotype of CYP2D6. This test diagnoses patients as extensive/normal (two copies of wild-type alleles), poor (one wild-type allele plus one variant allele with reduced enzyme activity or two variant alleles with reduced enzyme activity) and ultrarapid metabolizer (multiple copies of wild-type alleles). ${ }^{13)}$

The patient was genotyped as extensive (normal) metabolizer. As a result, elimination of aripiprazole involves two cytochrome P450 enzymes (CYP2D6, CYP3A4) and methylphenidate, which is both a substrate and a weak inhibitor of CYP2D6 at the same time, can lead to increased aripiprazole blood levels by blocking the metabolism of aripiprazole or by competing with aripiprazole for CYP2D6 metabolism. Thus, aripiprazole can cause hiccup as seen in our case.

In conclusion, it is recommended that clinicians should take drug interactions into consideration when using more than one drug concurrently. Profiling cytochrome P450 isoenzymes genotypes should be conducted in all patients prior to the onset of treatment when more than one drug is used in the same patient in order to benefit from treatment in the short term and to prevent side effects and drug 
interactions.

\section{REFERENCES}

1. Chang FY, Lu CL. Hiccup: mystery, nature and treatment. $J$ Neurogastroenterol Motil 2012;18:123-130.

2. Hori H, Nakamura J. Hiccups associated with switching from olanzapine to aripiprazole in a patient with paranoid schizophrenia. Clin Neuropharmacol 2014;37:88-89.

3. Kutuk MO, Tufan AE, Guler G, Yildirim V, Toros F. Persistent hiccups due to aripiprazole in an adolescent with obsessive compulsive disorder responding to dose reduction and rechallenge. Oxf Med Case Reports 2016;2016:66-67.

4. American Psychiatric Association. Diagnostic and Statistical Manual of Mental Disorders, Fifth Edition (DSM-5). Arlington, VI:American Psychiatric Publishing;2013.

5. Naranjo CA, Busto U, Sellers EM, Sandor P, Ruiz I, Roberts EA, et al. A method for estimating the probability of adverse drug reactions. Clin Pharmacol Ther 1981;30:239-245.

6. Nevels RM, Dehon EE, Alexander K, Gontkovsky ST. Psychopharmacology of aggression in children and adolescents with primary neuropsychiatric disorders: a review of current and potentially promising treatment options. Exp Clin Psychopharmacol 2010;18:184-201.
7. Nevels RM, Weiss NH, Killebrew AE, Gontkovsky ST. Methylphenidate and its under-recognized, under-explained, and serious drug interactions: a review of the literature with heightened concerns. German J Psychiatry 2013;16:29-42.

8. Berger P, Janowsky A, Vocci F, Skolnick P, Schweri MM, Paul SM. [3H]GBR-12935: a specific high affinity ligand for labeling the dopamine transport complex. Eur $J$ Pharmacol 1985;107:289-290.

9. Wall SC, Gu H, Rudnick G. Biogenic amine flux mediated by cloned transporters stably expressed in cultured cell lines: amphetamine specificity for inhibition and efflux. Mol Pharmacol 1995;47:544-550.

10. Maréchal R, Berghmans T, Sculier P. Successful treatment of intractable hiccup with methylphenidate in a lung cancer patient. Support Care Cancer 2003;11:126-128.

11. Gregory GA, Way WL. Methylphenidate for the treatment of hiccups during anesthesia. Anesthesiology 1969;31:89-90.

12. Tiwari AK, Souza RP, Müller DJ. Pharmacogenetics of anxiolytic drugs. J Neural Transm (Vienna) 2009;116:667677.

13. Müller DJ, Kekin I, Kao AC, Brandl EJ. Towards the implementation of CYP2D6 and CYP2C19 genotypes in clinical practice: update and report from a pharmacogenetic service clinic. Int Rev Psychiatry 2013;25:554-571. 